\title{
A Novel Solution for Sleep Scheduler in Wireless Sensor Networks
}

\author{
Harsh Kumar Singh, Jyoti Bharti \\ Department of Computer Science \& Information Technology \\ MANIT \\ Bhopal, India \\ Email: \{harshku4, jyoti2202\}@gmail.com
}

\begin{abstract}
.
A novel strategy is proposed to manage the sleep of the nodes in the network so that energy can be conserved and network connectivity can be kept. The novelty of the strategy is its extreme simplicity. The existing Gossip Based Sleep Protocol (GSP) is extended to achieve network reliability through piggybacking. Piggybacking is a convenient and effective approach and has been successfully applied to several areas of the networking. In the proposed work, we will develop a sleep management protocol and network reliability for wireless sensor networks. Then the protocol will be extended to the synchronous network, where nodes manage their own mode (sleep or awake mode) independently.
\end{abstract}

\section{Keywords}

sensor network; battery power; energy efficient routing; network reliability;

\section{Introduction}

Sensor network form a class of ad-hoc network, where the nodes are having basically low-cost, limited computing power that operates using batteries. They are deployed in a very huge numbers to collect data about the surroundings or any physical event like an aggregate information, and communicate parameters of interest to monitor nodes either on demand or as required. Typical scenarios of interest include seismic monitoring, power plant or nuclear reactor, traffic management, close-circuit camera in retail, military usage to sense the enemy territory. The nodes are organized into hierarchical cluster to reduce long range message transfer. To optimize power consumption message are short and bursty in nature, and spaced apart.

A good sensing coverage and connectivity at the same time can be provided in dense sensor nodes by keeping only necessary set of sensor nodes active to incerease lifetime of a network. While keeping other nodes in sleeping modes the scheme tries to not adversely affect the sensing coverage and connectivity. 
For each period the nodes which are having with lower remaining energy level are given higher priority which is done periodically for some selected sleep mode. So as to prolong the life time of the sensor nodes energy consumption load are more eventually distributed at the end of data transfer we find out that the energy level of nodes are distributed in randomly fashion as a result there is no single point of failure which eliminate exhaust condition from network.

\section{Related Works}

For collecting and communicating with some necessary nodes while keeping remaining modes into a sleep mode is the one way energy consumption. GAF [12] (Geographic adaptive fidelity). The networks are divided into grids. So nods are placed on the adjacent of a rectangular grid and each node can successfully communicate information. Here it is to be noted that at point time, only one node in each grid is active. GPS or other positioning system is required to gate the location information for grid formation.

SPAN [6] forms a multi-hop forwarding back bone to preserve the original capacity of the network. While remaining nodes can go to sleep mode so as to preserve energy. Back bone functionality is rotated amount the nodes to balance the energy consumption.

AFECA [5] define three operating states of nodes, sleeping, listening and active. Initially notes are in sleeping state. After Ts time period, it switches to the listening states, during the state that is listening state the radio turns on and listens for a message for a time period. If routing message is received during the period then node is active for routing. Here it change to the active stage if it decided to sent date or else as soon as the time period T1 space elapsed it will go to the sleeping state. AFECA algorithm uses the advantage of interchanging activates among nodes in a dense network. As densely increase so as to increase the life time.

This approach causes less complexity and lower energy consumption [2], but it has some disadvantage: 1) Network reliability is hard to preserve: 2) Synchronization cannot be forwarded to base station; 3) Optimization can be more difficult. A challenging jobs that is needed to consider and this paper tells the optimal solution for all the above problems Wireless sensor network are tiny and in expansible sensor node which are having small memory, computing power and work with batteries which work with the aid of batteries.

\section{Proposed Work.}

The objective of the proposed protocol is to look up overall network reliability by allowing nodes in the network to sleep for random time --proportional to their remaining battery life with a given gossip probability, with informing their neighbors or any control packet transmissions. A node tosses a coin to decide whether or not to forward a message. The probability $\mathrm{p}$ that a node forwards a message is called gossip probability. Period for which particular nodes are made active. The piggybacking on acknowledgement scheme is explicitly designed to be used in sensor network where data are usually flow form all the sensor to the monitor, which is a fixed node with greater computing and good power sources.

We assume that a single base station is placed in a random position inside the region. At the beginning the algorithm runs for every period to send control message for synchronization. 
Synchronization makes all nodes in awake mode. For each node to choose either going to sleep mode with probability $p$ or stay awake with probability $p=(1-p)$ for given period. At the end of each period all sleeping nodes wakeup so as to start for next period. Suppose source node send control message to its neighbor and return with maximum remaining energy of nodes through awake nodes. So from this process route is establish from one node to another node similarly this process also continue to its nearby nodes till destination node reached. When control message send to its neighbor with this piggybacking on acknowledgment is also used for synchronization. For any intermediate node to relay synchronization it will check maximum piggyback wait if it is not true than it send control message for relay synchronization if it is found to be true than generated piggyback which included control message and data which relay synchronization so as to send to next node for route discovery. To every end of period send all nodes receive control message so as to synchronize for next period.

$\begin{array}{lllll}1 & 2 & 3 & 4 & 5 \\ 6 & 7 & 8 & 9 & 10 \\ 11 & 12 & 13 & 14 & 15 \\ 16 & 17 & 18 & 19 & 20 \\ 21 & 22 & 23 & 24 & 25\end{array}$

Given- Form 1 to 25 are the nodes, Sources- Node 1, Destination- Node 20.

In this above $5^{*} 5$ matrix we are moving form source to destination. When node move form node 1 then to next node then it send control message so that all node get synchronized than after to start the algorithm of GSP+AODV where when moves it send the control packets so that one must give their remaining energy out of this given energy we choose one with highest energy as we select this condition than we move to neighbor node than its nearby node also repeat process which continue to nearby nodes so as to end up with destination node. While we move from one node to other we also add piggyback message so as we do route discovery we also collect the data form source to destination this process done through AODV protocol.

The node which is blue marked is best option to reach the destination node but as time progress this nodes will be exhaust because of this it is unreliable to send data. So to solve this problem we uses only that node which has highest energy so that above condition never comes or second method where we take grid topology the one with source node its size is taken than we take destination node size in above case it is 5,4 this size given for route discovery as soon as when from source node it move to next node it consider the one with not used often in this way it can reach to row no 4 but as soon as it move to row 5 than this size will be consider so as not to move beyond path in this way we can able to reach the destination node to show this path green marked 
International Journal Of Advanced Smart Sensor Network Systems ( IJASSN ), Vol 2, No.1, January 2012

\section{Results}

\subsection{Simulation Model}

The proposed synchronization protocol was implemented on NS2 Network simulator platform. To study the characteristic of GSP the distribution coordination function (DCF) of IEEE 802.11(b) for wireless LANs is used as the MAC/PHY layer. We have chosen a simulation area of $1500 \mathrm{~m} * 1500 \mathrm{~m}$ with number of nodes ranging from 10 to 120 . The Beacon Wait duration was taken $4^{*}$ slot where the slot duration for each slot width is $40 \mathrm{~ms}$. Also, the Maximum-PiggybackWait duration was taken in the range $1 \mathrm{~ms}$ to $100 \mathrm{~ms}$. The packet size was kept at 80 bytes. We used constant bit rate (CBR) as traffic source with average packet rate 0.5 packet/sec. The receiver and transmitter power were kept at $0.3 \mathrm{~mW}$. The simulations were run for $300 \mathrm{sec}$ to different source-destination traffic pair and various node positioning.

\subsection{Performance Result}

We compare the performance of our GSP+AODV protocol with it. We have taken parameters of our GSP+AODV protocol with it. We have taken three performance metrics (i) routing overhead. (ii) Consumed node energy of all the nodes after simulation and (iii) Delay which include looping error.

\section{Node Energy}

The consumed node energy of all routers at the end of simulation in Fig1.The graph shows that GSP+AODV has distributed overall energy over the entire network in a more balanced way, only the necessary node is kept active and remaining in sleep mode.

\section{Routing overhead for Piggyback}

The synchronization control information was piggybacked onto the data packet, being sent from the nodes. The routing overhead shown in Fig. 2 shows the characteristic of GSP+AODV have a better bandwidth as compared with AODV. The overall average bandwidth of GSP+AODV is $93 \%$ and AODV is $88 \%$. This better GSP+AODV come because of the presence of higher node energy network. It implies that routing overhead will improve than as a result bandwidth will improve and utilization of this bandwidth can be use for packets delivery. This is because while we send a message to synchronize we used a piggybacking which uses a lower level for timestamp as a result overhead decreases.

\section{Packet Network Delay:}

Packet delay variation (PDV) is the difference in end-to-end delay between selected packets in a flow with any lost packets being ignored. On using a same path again there is possibility of network failure as a result packet delay. So to counter-attack such problem higher node energy is uses to send the data from source to destination. On the other hand to overcome packet delay problem acknowledgment is used so as to minimize delay. 


\section{Remaining Energy of Nodes}
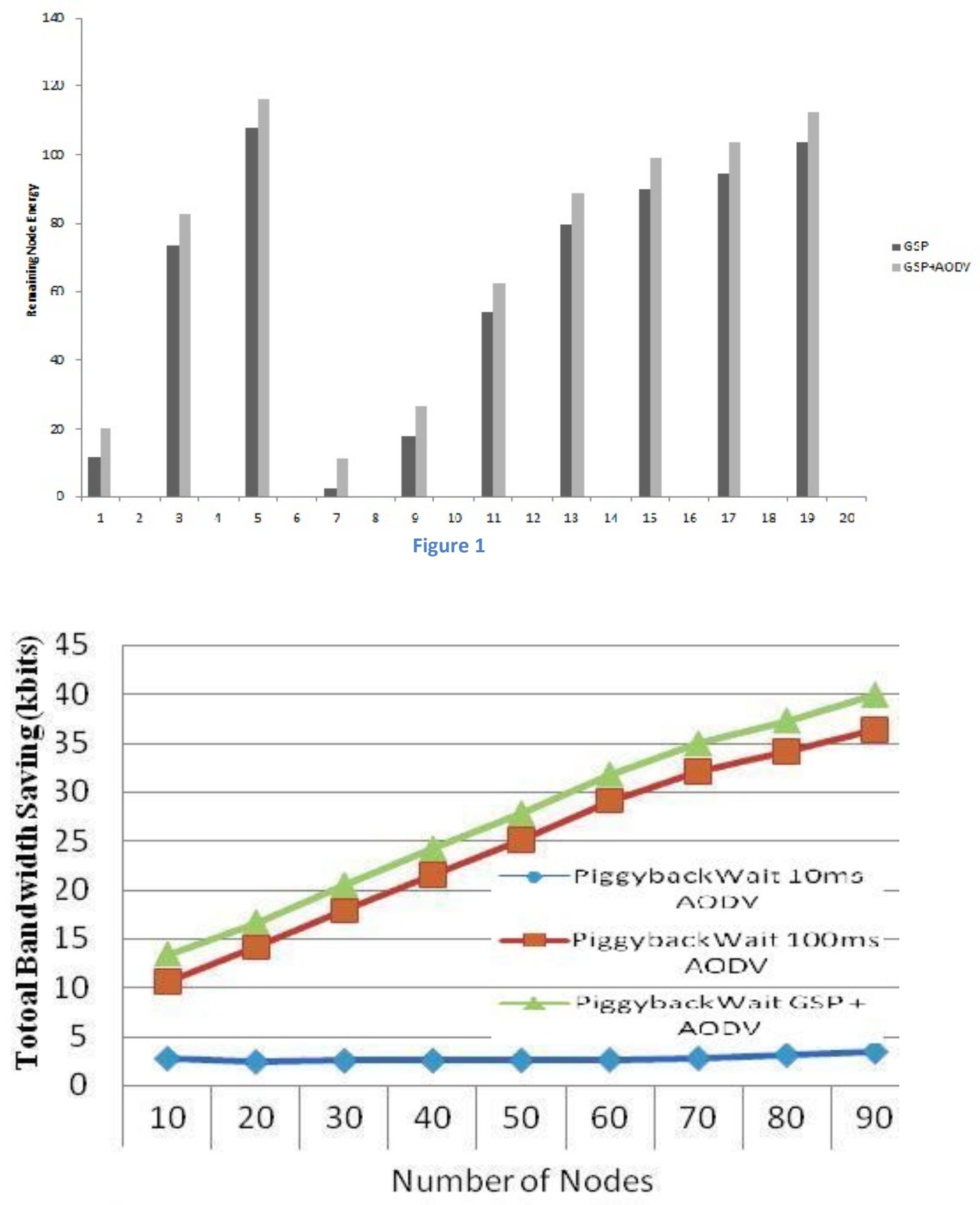

Figure 2

\section{Conclusion \& Future Work}

Our research state due to this protocol ie GSP energy consumption is more evenly distributed in the entire network since the average energy of the network decrease because the traffic forwarding continuously via the same path can be avoided. To address various properties of GSP, e.g. With GSP using energy cosumption the network is fully reliable since entire nodes go to 
sleep in fully randomly fashion. So as a result there is no single point of failure or backbone infrastructure that can fail and therefore there is higher reliability. We are evaluating network lifetime improvement along with other standard metrics to find out effectiveness of the proposed scheme as compared to the existing ones. Deriving optimal values of protocol parameters for a given network configuration. Deriving optimal link of protocol parameters for given network configuration when both source and destination nodes is static so as to increase reliability is part of future work.

\section{References}

[1] Xiaobing Hou, Tipper, D., Gossip-based sleep protocol (GSP) for energy efficient routing in wireless ad hoc networks, in IEEE WCNC 2004, 21-25 March 2004 Page(s): 1305 - 1310 Vol.3.

[2] E. Bulut, I. Korpeoglu, A Dynamic Sleep Scheduling Protocol for prolonging the Lifetime of Wireless Sensor Networks. 21 st International Conference on AINAW'07,0-7695-2847-3/07, 2007.

[3] F. Ye, G. Zhong, S. Lu and L. Zhang. PEAS: A Robust Energy Conserving Protocol for Long-lived Sensor Networks, in the 23rd International Conference on Distributed Computing Systems, 2003.

[4] F. Ye, S. Lu, and L. Zhang.GRAdient Broadcast: A Robust, Long-lived Large Sensor Network. http://irl.cs.ucla.edu/papers/grab-tech-report.ps, 2001.

[5] Y. Xu, J. Heidemann and D. Estrin. Adaptive Energy Conserving Routing for Multihop Ad Hoc Networks. USC/ISI Research Report 527, 2000.

[6] B. Chen, K. Jameison, H. Balakrishnan, and R. Morris.SPAN: An Energy Efficient Coordination Algorithm for Topology Maintenance in Ad Hoc Wireless Networks.MOBICOM 2001.

[7] D. Tian, and N. Georganas A Coverage-Preserving Node Scheduling Scheme for Large Wireless Sensor Networks, Proceedings of the 1st ACM international workshop on Wireless sensor networks and applications, Atlanta, Georgia, pages: 32 - 41, 2002.

[8] W. Heinzelman, A. Chandrakasan, and H. Balakrishnan, Energy-Efficient Communication Protocol for Wireless Sensor Networks. Proceedings of the Hawaii International Conference on System Sciences, 2000.

[9] O. Kasten. Energy consumption, http://www.inf.ethz.ch /kasten/research/bathtub/ energy consumption.html.

[10] W. Ye, J. Heidemann, D. Estrin. An Energy Efficient MAC Protocol for Wireless Sensor Networks. IEEE Infocom, 2002.

[11] I.F. Akyildiz, W. Su, Y. Sankarasubramaniam,E. Cayirci, Wireless Sensor Networks: A Survey,Computer Networks, 2002.

[12] Y. Xu, J. Heidemann \& D. Estrin. Geography Informed Energy Conservation for Ad Hoc Routing. MOBICOM, 2001.

Bibliography

Harsh Kumar Singh received the B.E. degree in Information Technology from RGPV University, Bhopal, India, in 2006, and the M.TECH degree in Computer Science \& Engineering from MANIT University, Bhopal, India, in 2011. His current research interests include wireless sensor networks, Time Synchronization

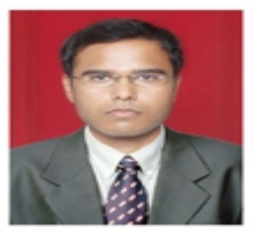

Jyoti Bharti born in 1976, obtained Bachelor of Engineering degree in Computer Science and Engineering in 2000 from Lakshmi Narain College of Technology affiliated to Barkatullah University Bhopal. Did Masters of Technology from Samrat Ashok Technical Institute Vidisha affiliated to Rajiv Gandhi Proudyogiki Vishwavidyalaya Bhopal, MP, India. Area of research is Image processing and Data mining. Total teaching Experience is more than 9 years. After serving 3 years in Lakshmi Narain College of 
International Journal Of Advanced Smart Sensor Network Systems ( IJASSN ), Vol 2, No.1, January 2012

Technology and RKDF Institute of Science and Technology Bhopal, MP, India, joined the Department of Information Technology, Maulana Azad National Institute of Technology, and Bhopal as lecturer. Currently serving the Institute as Assistant Professor in Department of Information Technology. Persuing the $\mathrm{PhD}$ work in the area of Image processing. Published more than 7 papers in various National $\backslash$ International conferences,

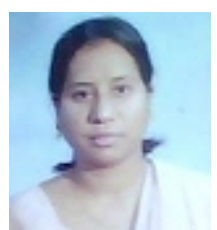
journals and has attended more than 10 short term courses on Image processing, Data mining, and Security. 